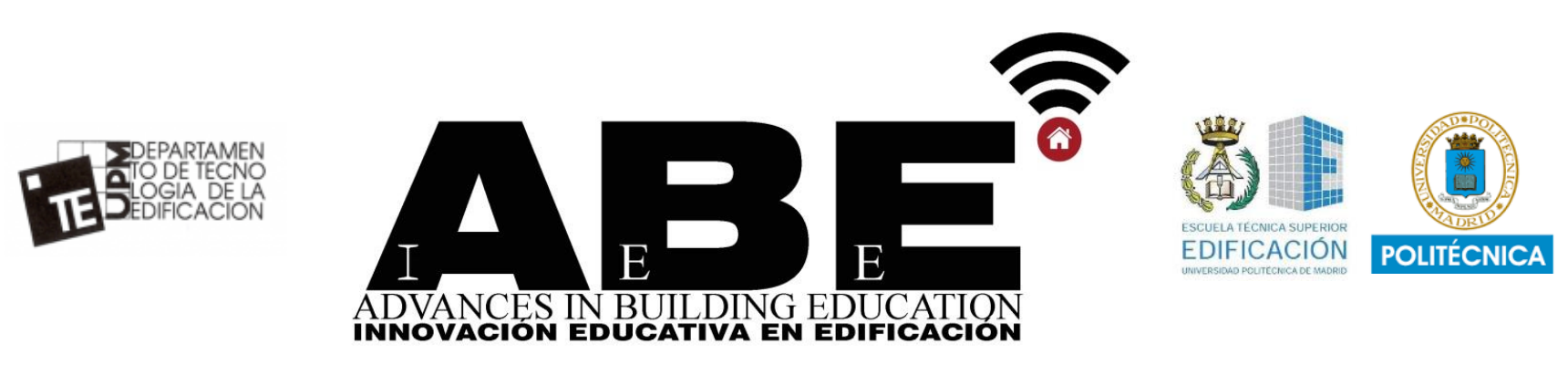

\title{
Innovación docente y sostenibilidad en el aula
}

\author{
Innovation and sustainability in the classroom
}

\author{
Belén Andrés Segovia ${ }^{*}$ \\ $1^{*}$ Doctora en Derecho por la Universitat de València \\ 2 Profesora asociada en Derecho Administrativo de la Universitat Jaume I de Castellón. \\ Correo: andresb@uji.es
}

\section{Recibido: 30/11/2021 | Aceptado: 01/12/2021 | Fecha de publicación: 01/12/2021} DOI:10.20868/abe.2021.3.4733

\section{TITULARES}

- La incorporación de las TICs y la sostenibilidad en el aula.

- Aprende, difunde y vuelve a aprender.

- Necesitamos construir un nuevo marco educativo, y pronto

- Los avances tecnológicos, la sostenibilidad y las realidades educativas, configuran nuestro presente y nuestro futuro

\section{HIGHLIGHTS}

- TICs and sustainability implementation in the classroom.

- Learn, spread and learn again.

- We need to built a new education framework, and soon.

- Technological advances, sustainability and education realities, shaping our present and our future 


\section{RESUMEN}

El trabajo que presentamos tiene por objeto aprender y debatir sobre cómo la experiencia digital va acusándose ante un sistema educativo que se torna volátil y cambiante. El papel de los actores que participan en este nuevo escenario que ofrece internet es clave puesto que de los mismos dependerá su uso: responsable, crítico, respetuoso y creativo. La senda pasa pues, por crear formulas emergentes en el aula que no solo apuesten por un progreso inteligente sino que además requiere que sea inclusivo y sostenible. Estos elementos, constituyen los pilares sobre los que se cimentarán las nuevas políticas públicas de la Comisión Europea. Visto en positivo, no debemos olvidar que, la mejor forma de potenciar la calidad del medio ambiente pasa por implicar a todos los ciudadanos y la educación nos brinda una excelente oportunidad para lograrlo.

Palabras clave: Digitalización; Innovación Docente; Sostenibilidad; Educación.

\section{ABSTRACT}

This communication proposal offers the opportunity to learn about and discuss how the digital experience is becoming increasingly evident in a education system that is becoming, volatile and changing. The actors in this Internet scenario are the key, as its use will depend on them: responsible, critical, respectful and creative. The classroom demands that this progress be inclusive and sustainable. These elements constitute the pillars on which the European Commission's new public policies. On a positive note, we must not forget that the best way to improve the environment is to involve all citizens, and education provides an excellent opportunity to achieve this.

Keywords: Digital; Teaching Innovation; Sustainability; Education.

\section{INTRODUCCIÓN}

La Agenda 2030 para el Desarrollo Sostenible lanzó en 2015 una propuesta con el fin de poder alcanzar erradicar la pobreza y de este modo lograr un planeta más saludable que apuesta por la igualdad de oportunidades [2]. Sobre esta premisa debemos destacar que todos los alumnos son iguales pero unos son más iguales que otros. Las inquietudes que adquiere el propio alumno cuando se enfrenta a una clase de Grado, en cualquiera de las ciencias del saber, no siempre es homogénea, sino que dependerá de sus necesidades presentes y futuras. Véase por caso, bien la decisión de continuar su formación con la realización del doctorado o bien que le sirva de impulso en su lucha por obtener un puesto de trabajo en el mercado laboral. Sin embargo, con independencia de los objetivos que planteen, todos ellos convergen en un interés común que es la obtención de una formación de calidad. Es en este aspecto donde algunas de las 17 metas suscritas por las Naciones Unidas cobran un papel protagonista. Su tenor, invita a los diferentes países a que lleven a cabo una transformación social, económica y cultural sin parangón que servirá para garantizar los derechos humanos de todos.

Los docentes se ven compelidos a adaptar las clases tradicionales a nuevos modelos que adquieren un impacto global y que servirán para construir una sociedad más inteligente y sostenible. En estos términos, se ha pronunciado la meta 4.7. por cuanto apuesta por un sistema educativo de calidad, señalando que de aquí a 2030 , se deberá 
"asegurar que todos los alumnos adquieran los conocimientos teóricos y prácticos necesarios para promover el desarrollo sostenible, entre otras cosas mediante la educación para el desarrollo sostenible y los estilos de vida sostenibles, los derechos humanos, la igualdad de género, la promoción de una cultura de paz y no violencia, la ciudadanía mundial y la valoración de la diversidad cultural y la contribución de la cultura al desarrollo sostenible". Para su realización se precisa de una intensa voluntad política y docente que permita desarrollar una acción decidida que se presenta como ambiciosa dado el gran número de personas que se verán afectadas. Para su éxito, requiere del esfuerzo de todos. De lo contrario, como ya se advirtió en la Cumbre de los ODS celebrada en septiembre de 2020, los esfuerzos para lograr el cambio habrán sido insuficientes. Esta iniciativa, pone entre las cuerdas el compromiso adoptado en la Agenda y en la que se verán afectadas las generaciones presentes y futuras [3].

Con todo no hay nada perdido. La transformación digital nos ofrece herramientas óptimas con las que poder lograr liderar, movilizar y crear acciones que nos permitirá vencer no solo la problemática generada por la Covid-19 sino a su vez servirá para lograr concienciar sobre la importancia que adquiere la sostenibilidad en el sistema educativo. Es una era de cambios, el primero de ellos, se logra con el paso de los formatos presenciales a otros virtuales. El segundo, la ayuda de las plataformas digitales con el fin de facilitar la comprensión de las clases como mecanismo complementario a las mismas. En tercer lugar, el uso de la sostenibilidad como objetivo para lograr no solo que las universidades se adapten a los nuevos cambios sino que el modelo educativo impulse estrategias desde el aula. A este último supuesto es al que le dedicaremos una especial atención puesto que el mismo determinará el camino a seguir para mejorar el cambio climático, atender a la erradicación de la pobreza, las desigualdades, apostar por el empoderamiento de género y la creación de sociedades más inclusivas y equitativas.

Los alumnos/as no pueden ser ajenos a la realidad que ocupa y afecta a la sociedad en la que habitan. Marc Tucker señaló el desafío a los que se enfrentan los sistemas educativos que requieren de un aprendizaje profundo para todos los estudiantes y no solo para las máquinas. De este modo señaló que, "nuestros estudiantes deberán comprender las grandes ideas en las materias básicas del plan de estudios. Deberán tener una comprensión profunda de los conceptos subyacentes que estructuran el conocimiento en esas materias básicas. Tendrán que usar esos conceptos todos los días para resolver problemas complejos en dominios que encuentren interesantes e incluso convincentes" [4]. Incorporar en las estrategias docentes los factores que atiendan a los sostenibilidad significará brindar una serie de oportunidad para que los estudiantes puedan desarrollar sus habilidades haciendo uso de ideas nuevas que permitan desarrollar su pensamiento creativo y crítico que será favorable para resolver en un futuro los problemas reales. Los riesgos que se plantean en este ámbito quedan resumidos en el siguiente cuadro. 
Tabla 1: Datos extraidos del informe de la World Economic Fórum [5]

Véase en: http://reports.weforum.org/global-risks-report-2021/methodology/

\begin{tabular}{|c|c|c|}
\hline \multirow{7}{*}{ 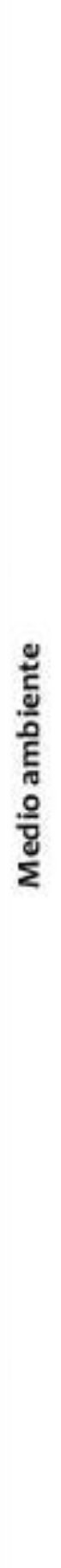 } & \multicolumn{2}{|r|}{ Riesgos globales 2021} \\
\hline & $\begin{array}{l}\text { Pérdida de biodiversidad y } \\
\text { colapso del ecosistema }\end{array}$ & $\begin{array}{l}\text { Consecuencias irreversibles para el medio ambiente, la } \\
\text { humanidad y la actividad económica, y una destrucción } \\
\text { permanente del capital natural, como resultado de la } \\
\text { extinción y/o reducción de especies. }\end{array}$ \\
\hline & $\begin{array}{l}\text { Fracaso de la acción } \\
\text { climática }\end{array}$ & $\begin{array}{l}\text { La incapacidad de los gobiernos y las empresas para hacer } \\
\text { cumplir, promulgar o invertir en medidas efectivas de } \\
\text { adaptación y mitigación al cambio climático, preservar los } \\
\text { ecosistemas, proteger a las poblaciones y hacer la transición } \\
\text { a una economía neutra en carbono. }\end{array}$ \\
\hline & $\begin{array}{l}\text { Eventos climáticos } \\
\text { externos }\end{array}$ & $\begin{array}{l}\text { Pérdida de vidas humanas, daños a los ecosistemas, } \\
\text { destrucción de bienes y / o pérdidas económicas a escala } \\
\text { global como consecuencia de eventos climáticos extremos: } \\
\text { frentes fríos, incendios, inundaciones, olas de calor, } \\
\text { vendavales, etc. }\end{array}$ \\
\hline & $\begin{array}{l}\text { Daño ambiental causado } \\
\text { por el hombre }\end{array}$ & $\begin{array}{l}\text { Pérdida de vidas humanas, pérdidas económicas y / o daños } \\
\text { a los ecosistemas como resultado de la actividad humana y } \\
\text { o falta de convivencia con los ecosistemas animales: } \\
\text { desregulación de áreas protegidas, accidentes industriales, } \\
\text { derrames de petróleo, contaminación radiactiva, comercio } \\
\text { de vida silvestre, etc. }\end{array}$ \\
\hline & $\begin{array}{l}\text { Grandes desastres } \\
\text { geográficos }\end{array}$ & $\begin{array}{l}\text { Pérdida de vidas humanas, pérdidas económicas y / o daños } \\
\text { a los ecosistemas como consecuencia de desastres } \\
\text { geofísicos: terremotos, deslizamientos de tierra, tormentas } \\
\text { geomagnéticas, tsunamis, actividad volcánica, etc. }\end{array}$ \\
\hline & $\begin{array}{l}\text { Crisis de recursos } \\
\text { naturales }\end{array}$ & $\begin{array}{l}\text { Crisis químicas, alimentarias, minerales, hídricas u otros } \\
\text { recursos naturales a escala mundial como resultado de la } \\
\text { sobreexplotación humana y / o la mala gestión de recursos } \\
\text { naturales críticos }\end{array}$ \\
\hline
\end{tabular}

Advances in Building Education / Innovación Educativa en Edificación | ISSN: 2530-7940 | http://polired.upm.es/index.php/abe

| Cod. 2103 | Septiembre - Diciembre 2021 | Vol. 5 № 3 | pp. 41/61 | 


\section{METODOLOGÍA}

El sistema educativo esta conectado con una perspectiva amplia de lo que supone la simple adquisición del conocimiento, lo que pone en valor el dónde y cuándo tendrá lugar el aprendizaje. Si bien es cierto que, como el propio título del trabajo indica, enmarcaremos el presente análisis en el 'aprendizaje en el aula', debemos destacar, de forma breve, que también existe la posibilidad de aprender fuera de la escuela. En estos términos, la resolución de los problemas reales, se suceden a diario en los diferentes sectores productivos.

La posición que ocupan los profesionales que trabajan en los diferentes gremios será observada por los estudiantes, empleando técnicas de innovación docente que apuestan por la formulación de juegos que les permita trabajar en equipo.
La motivación del alumnado en este campo es notoria. Con esta técnica, se podrán establecer metas que permitirán mejorar los objetivos establecidos en el plan de estudios, así como planificar nuevas técnicas para lograr motivar al público asistente a las clases desde la: creatividad, empatía, realizando prácticas sociales, desarrollando el pensamiento crítico, etc. El desafío para el docente, pasa por crear una metodología que de respuesta a los retos sociales y educativos. En concreto, cómo crear oportunidades y cómo importar la experiencia al aula de modo que los estudiantes aprendan que la redacción de un caso práctico puede ser igualmente válido para la superación de la asignatura como para su futuro laboral. Para atender a los citados criterios deberemos seguir el siguiente esquema:

\section{Resumen de la metodología a seguir para la propuesta de la práctica}

\begin{tabular}{|c|c|c|}
\hline & Investigación 1 & Investigación 2 \\
\hline $\begin{array}{l}\text { Duración: Métodos de } \\
\text { recopilación de datos }\end{array}$ & \multicolumn{2}{|c|}{ Enero-Mayo (segundo semestre) } \\
\hline $\begin{array}{l}\text { Criterios de selección } \\
\text { de grupos }\end{array}$ & \multicolumn{2}{|c|}{ Libre elección de grupos por parte de los estudiantes } \\
\hline \multirow{2}{*}{$\begin{array}{l}\text { Participantes } \\
\text { Áreas académicas }\end{array}$} & 5 alumnos del Grado en Derecho & 5 alumnos del Grado en Edificación \\
\hline & $\begin{array}{l}\text { 1) Búsqueda de fondos europeos } \\
\text { 2) Análisis de la aplicación de los } \\
\text { objetivos señalados en la Agenda }\end{array}$ & $\begin{array}{l}\text { 1) Estudio de materiales } \\
\text { 2) Búsqueda de estrategias basadas } \\
\text { en la sostenibilidad }\end{array}$ \\
\hline Roles & $\begin{array}{l}\text { España Digital } 2025 \\
\text { 3) Regulación en materia medio } \\
\text { ambiental } \\
\text { 4) Estrategias para suprimir la brecha } \\
\text { geográfica } \\
\text { 5) Regulación del Derecho Digital }\end{array}$ & $\begin{array}{l}\text { 3) Caracteristicas del lugar donde se } \\
\text { va a desarrollar la construcción } \\
\text { 4) Parte artistica: bocetos } \\
\text { 5) Análisis de los costes de la obra }\end{array}$ \\
\hline Análisis de género & \multirow{2}{*}{\multicolumn{2}{|c|}{$\begin{array}{l}\text { La constitución de los grupos deberá atender a criterios de igualdad } \\
\text { La propuesta de esta actividad será considerada como práctica de evaluación } \\
\text { continua y no deberá exceder el } \mathbf{4 0 \%} \text { de la calificación final. El resto de la } \\
\text { calificación se obtendrá tras la superación de un examen teórico que tendrá } \\
\text { una valoración que ocupará el } 60 \% \text { sobre la nota final. }\end{array}$}} \\
\hline Evaluación & & \\
\hline
\end{tabular}

\subsection{Premisas para la implementación de la} sostenibilidad en el ámbito educativo
Desde la Unión Europea se están comenzando a esbozar iniciativas que pretenden desarrollar el Espacio Europeo de Educación en torno a seis dimensiones, que serán muy útiles para 
comprender el objeto de la práctica que se propone.

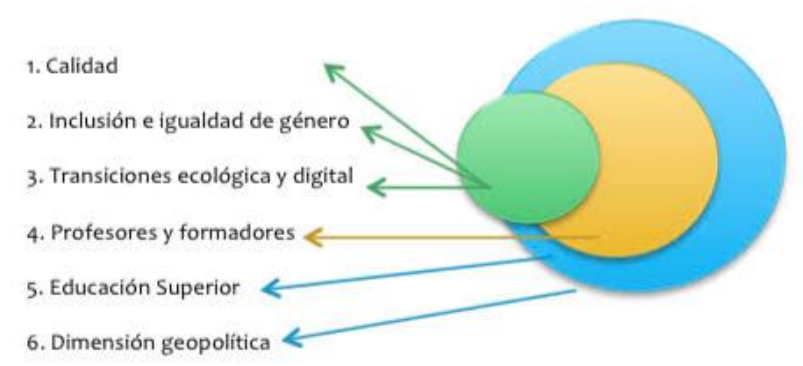

Tabla 3: Gráfico de elaboración propia [6]

Para lograr el éxito, en esta práctica se deberán considerar los 6 criterios señalados del siguiente modo. Desde el ámbito subjetivo, tenemos por un lado, la figura del evaluador. Es decir, profesores y formadores de educación superior, que a su vez guiarán a los estudiantes sobre cómo crear estrategias que les permitirá superar la tarea. Por otro lado, desde un enfoque más amplio, se observará cómo los alumnos aplican los conocimientos teóricos al ámbito práctico. De este modo, los docentes podrán conocer si los estudiantes han desarrollado y considerado las estrategias señaladas como metas en los Objetivos de Desarrollo Sostenible en la Agenda 2030 de las Naciones Unidas. A modo de ejemplo, la garantía de inclusión e igualdad de género en sus proyectos. Por último, desde una dimensión técnico-jurídica se valorará el contenido. En concreto, la creatividad y el análisis crítico que los estudiantes desarrollen en la aplicación y presentación de sus proyectos atendiendo a: la transición ecológica y digital (conjunta), la calidad de la construccción (Grado en Edificación), la dimensión geopolítica (Grado en Derecho). Todos estos aspectos serán estudiados con detenimiento a continuación.

\subsection{Supuesto práctico para la convergencia de las 6 estrategias}

La realización de una práctica formativa que guarde relación con la sostenibilidad pretende observar y dar respuesta a la cuestión sobre cómo sería la materialización del cumplimiento de dos de las bases sobre las cuales se cimentará la política europea: la digitalización y el Pacto Verde Europeo [7]. Aunque la actividad, con carácter primigenio, estaba dirigida a los alumnos/as de Derecho se prevé que su propuesta conjunta con los estudiantes del Grado en Edificación sea muy positiva. Al fin y al cabo, el mundo laboral se enriquece con la colaboración público-privada de los diferentes agentes que participan del mercado. Para reforzar esta afirmación, podemos observar cómo estos acuerdos entre sector público y sector privado se han visto potenciados con la crisis sanitaria actual. Las debastadoras consecuencias que ha provocado la Covid-19 en los diferentes países hace que desde la Unión Europea se prevean un conjunto de fondos europeos con los que se pretende impulsar la recuperación económica de los diferentes Estados miembros: el Marco Financiero Plurianual (2021-2027) y los Fondos Next Generation EU (2021-2024) [8].

Con vistas a esta situación, el Gobierno de España busca aprovechar la oportunidad que brindan las instituciones europeas con el fin de liderar el mercado digital y apostar por la lucha contra el cambio climático. Ante esta inminente transición tecnológica y verde se ha aprobado la Agenda España Digital 2025, en la que quisiera hacer enfásis en el séptimo de sus objetivos, por cuanto consiste en lograr que "Acelerar la digitalización del modelo productivo mediante proyectos tractores de transformación digital en sectores económicos estratégicos (...). Estos proyectos tienen como meta una reducción del $10 \%$ de las emisiones de $\mathrm{CO} 2$ por efecto de la digitalización de la economía en 2025" [9]. La colaboración de sendos sectores servirá para alcanzar estos objetivos con mayor eficacia.

La propuesta que realizamos, como observaremos en los siguientes apartados, pretende crear sinergias entre ambas titulaciones. Los resultados que se obtengan pueden constituir el inicio de futuros proyectos tractores que competirán por la 
obtención de ayudas públicas que les será muy útiles para sufragar los costes de su implementación. Centrandonos en el caso práctico, debemos seañalar la redacción del siguiente supuesto práctico.

"Cada equipo, forma parte de una empresa contructura que desea concurrir a un proceso de selección para la creación de una oficina de turismo en Chodos, localidad rural situada en Castellón de la Plana, España. Con su construcción lo que se pretende lograr es incentivar a la ciudadanía a que apuesten por una nueva modalidad de turismo rural y evitar casos como los que plantea la España vaciada. El Ayuntamiento realiza una convocatoria pública -que será diseñada por el docente- en la que abre un periodo para que las diferentes empresas/grupos muestren sus propuestas para la contrucción de la obra. Todas ellas serán consideradas en el proceso de selección y será elegidas aquellas que más se ajusten a los principios de: eficacia, sostenibilidad y precio de mercado".

\subsection{Sujetos participantes de la tarea}

La estructutra del caso práctico que ofercemos en el presente estudio, crea las siguientes categorías de actores o sujetos que serán esenciales para realizar con éxito la actividad: alumnos (del Grado en Derecho y Grado en Edificación) y los docentes. Las tareas que les serán asignadas son heterogeneas pero interdependientes las unas de las otras:

a) Los primeros de ellos, lo conforman los estudiantes del Grado en Derecho. A ellos compete la labor de destinar la información jurídico-política que desde Europa y desde el Gobierno de España se tenga en consideración para que los proyectos tractores ajusten sus obras a las necesidades actuales del mercado. En ese sentido, su labor pasa por definir cómo se llevará a cabo la prestación de los servicios con el objeto de dar cumplimiento a los tres factores determinantes en la priorización de las diferentes ofertas públicas: sostenibilidad, digitalización y brecha georgráfica.

b) El segundo grupo está compuesto por los alumnos del Grado en Edificación. La tarea que se les asigna en este proceso pasa por crear bocetos que servirán para la prestación del servicio y que permitirán observar las características de los planos, físicos o virtuales, que serán defendidos en la oferta pública. Aunque las diferentes acciones pueden ser llevados por un mismo sujeto que tenga conocimientos de ambas materias, por regla general la especialización de sendos campos, jurídico-técnico, se encuentra diferenciada. La participación de los dos perfiles de alumnos permitirá determinar, con mayor rigor, los diferentes aspectos que envuelven a un proyecto ante una oferta pública.

c) El tercer participante de este proceso son los docentes. Tanto el responsable de la asignatura en el Grado en Derecho como el que ostenta el cargo en el Grado en Edificación se convierten en intermediarios o mediadores en el proceso. Competerá a los mismos: 1) diseñar la convocatoria pública que deberá guiar las prácticas; 2) resumir las diferentes ofertas; $y, 3)$ examinar los parámetros de calidad y el cumplimiento de las políticas públicas. En definitiva, replicar un proceso de selección que permita dirigir los conocimientos teóricos adquiridos en las clases a la práctica. Asimismo, deberá atender a las dudas que planteen los alumnos a través de un foro que se habilitará en la propia plataforma donde se desarrollará la tarea.

De todas estas categorías, la resolución del caso práctico descansa en las dos primeras. La posición de mediador que se le otorga al docente tiene consecuencias muy notables pero de él no depende la resposabilidad final de su ejecución. Sin embargo, su papel si que servirá para poder atender de forma personalizada a las inquietudes que planteen los estudiantes y sobre él mismo recaerá la labor de fomentar el pensamiento: crítico, creativo, responsable y respetuoso con el 
medio ambiente. Señalados estos motivos, a continuación nos detendremos en analizar sus perfiles e indicaremos el régimen al que obedece su responsabilidad.

\subsubsection{El papel de los estudiantes de Grado en Derecho}

Para entrar en el contexto en el que se enmarca la tarea, debemos destacar que la actividad va dirigida a un grupo de alumnos/as de la asignatura de Derecho Administrativo (asignatura obligatoria en el Grado en Derecho). También podría ser aplicable en asignaturas optativas tales como el Derecho urbanístico, presente en el Plan de Estudios de algunas universidades. Entre las materias que se abordan en sendas asignatura se encuentra el estudio que versa sobre la contratación pública.

\subsubsection{El papel que ocupan los alumnos/as del Grado en Edificación}

La construcción es un sector fundamental que trabaja para lograr la creación de productos únicos que combinan las necesidades humanas y las características del territorio que habitamos. Los especialistas en el Grado en la Edificación moldean las ciudades y zonas rurales, así como las estructuras que las abastecen y conectan, acorde a las necesidades que plantea cada época. De este modo, permiten heredar las ciudades del pasado y construir las nuevas infraestructuras del futuro. En el proceso intervienen agentes que forman parte de los diferentes sectores. En el caso de los estudiantes del Grado en Edificación se convierten, a través de la construcción empleando materiales tales como la madera, metal, energía, telecomunicaciones, ect., en una industria de industrias que persigue el desarrollo de las infraestructuras que serán el centro de las actividades humanas. Es importante señalar por tanto, que las construcciones guardan una estrecha relación con el lugar donde se encuentra situada la obra. En consecuencia, para lograr su éxito deberán atender a: por un lado, una perspectiva georgráfica, ya que el lugar donde irá ubicada la obra condicionará su construcción; por otro lado, una vez finalizada la obra, la perspectiva que adquiere su impacto determinará el contexto, y, finalmente, cómo la revolución tecnológica facilita la creación de nuevas estructuras consiguiendo una mayor eficacia y calidad, sin descuidar su objeto. Por todo lo señalado, esta actividad puede ser una propuesta que lleven a cabo alumnos de asignaturas tanto obligatorias como optativas. A modo de ejemplo podemos citar los estudiantes de una asignatura de tercer curso del Grado, como pudiera ser Construcción sostenible (obligatoria) en el Plan de Estudios del Grado en Edificación de la Universidad Politécnica de Madrid, o los que hayan escogido cursar la asignatura de Control de la edificación y desarrollo sostenible (optativa), presente en el Plan de Estudios de algunas instituciones como la Escuela tecnica superior de Ingeniería de la Edificación en Granada, dentro del Grado en Edificación. La participación de los estudiantes de esta rama técnica permite dar cuenta de cómo la industria de la construcción cambia su modelo productivo hacia formatos más inteligentes y sostenibles. Para ello, deberán aprovecha las virtudes que ofrece la industria 4.0. que surge con motivo de la que se conoce como la Cuarta Revolución Industrial [10].

\subsubsection{El papel de los profesores y formadores}

En cualquier proceso que se aprecie que intente realizar una apuesta por la innovación docente no debe descuidar el papel que ocupa los profesores y formadores. EI empleo de herramientas tecnológicas no puede prescindir de la figura del maestro. La máquina debe suponer, en todo caso, una ayuda que le permita obtener un mayor rendimiento y satisfacción en la tarea que le ha sido otorgada, enseñar -y aprender-. Por ello, debemos comenzar destacando que las figuras del profesor y del alumno no podrán ser sustituidas por las nuevas tecnologías sino que les permitirá potenciar la creatividad y el 
ingenio ante los nuevos retos que planteen las materias objeto de estudio. Su objeto pasa por educar en el sentido primigenio del término. En concreto, está llamado no solo a transmitir los conocimientos sino tambien a: dirigir, encaminar, perfeccionar y desarrollar el proceso de aprendizaje. Todo ello sin descuidar las particularidades que presenten las facultades morales e intelectuales propias de cada centro.

\subsection{Proyectos interesantes que pueden elaborar los estudiantes}

Uno de los factores que motivan el presente trabajo colaborativo pasa por la construcción de un plan que potencie el mercado ante los nuevos retos a los que se exponen los Estados miembros. La práctica deberá tener cabida en las diferentes directrices que se marcan desde la Unión Europea. En este sentido, adquiere un valor especial aquellas aportaciones que realicen los alumnos al proyecto que presenten y que tenga en cuenta cada uno de los ejes estratégicos señalados. El docente, será el encargado de coordinar: las bases que deberán presidir la redacción de las prácticas, la selección de los grupos, la dotación que se ofrece a cada uno de los trabajos. Señalados estos aspectos, uno de los consejos que se ofrece a los alumnos es que tengan en cuenta que la apuesta por un proyecto transformador, desde el punto de vista tanto instucional como europeo, puede suponer grandes ventajas en el proceso competitivo puesto que podrán optar a subvenciones que de otro modo no llegarían a materializarse. Resulta fundamental pues, que los estudiantes del Grado en Derecho informen a los alumnos del Grado en Edificación sobre la evaluación de los criterios, requisitos y la redacción de planes estratégicos que permitirán potenciar sus proyectos. Compete, por otro lado, a los alumnos del Grado en Edificación observar la viabilidad de los proyectos y observar si es posible materializar algunas de las premisas señaldas desde la Unión Europea anunciadas por sus compañeros de la vertiente jurídica. En definitiva, lo que la presente práctica pretende es que los alumnos sean conscientes de cómo el trabajo en equipo puede resultar beneficioso para sus intereses empresariales y cómo sus decisiones pueden tener un fuerte impacto sobre la sostenibilidad. Para su consecución, existen cuatro elementos que resultan claves para lograr un proyecto tractor atractivo como oferta a presentar en la práctica: la digitalización, la sostenibilidad, la recuperación del medio rural y la brecha de género.

\subsubsection{Proyectos enfocados a la construcción y la digitalización}

Entre las opciones que harán atractiva una propuesta, como la planteada, de proyecto para la construcción de una oficina de turismo, encontramos el desarrollo de estrategias que incorporan herramientas digitales en sus negocios. La transición digital constituye junto con la sostenibilidad dos de los factores que las empresas conciben como una oportunidad de que sus trabajos lideren las ofertas públicas generando valor en su implementación. Su importancia ha sido resaltada por la Comisión Europea por cuanto lo sitúa como un enclave para lograr una mayor competitividad con independencia del tamaño que adopte la empresa o el sector en el que tenga lugar su incorporación. La complejidad que adquiere la construcción de estas iniciativas se ve compensada por las múltiples ventajas que en el corto plazo pueden adoptar. Véase por caso: una mejora en los ingresos, ahorro de costes, procesos automatizados, eficientes y escalables, etc. Estas habilidades requieren de una especialización del personal que participará del proyecto, por lo que permitirá un aumento y progresión en la generación de empleo. En suma, son muchas las virtudes que presentan apostar por el diseño de bocetos que atiendan a estas características, ya que favorecerá la consecución de dos elementos: mejorar sus ofertas y sobrevivir al modelo económico actual.

\subsubsection{Proyectos relacionados con la sostenibilidad}


Los alumnos/as deberán presentar un proyecto de sostenibilidad que encaje con los objetivos estratégicos señalados desde Europa y el Gobierno de España. La existencia de fondos destinados a la recuperación económica generada por la Covid-19, obedece a tres motivos:

La importancia que adquiere estos temas sobre la sociedad. Las empresas del futuro deberán incorporar, a sus estrategias, factores que favorezcan la sostenibilidad, de lo contrario corren el riesgo de ver deteriorada su reputación y relevancia comercial.

Las empresas que lo apliquen dispondrán de incentivos fiscales para su consecución. Con esta iniciativa se pretende lograr que la apuesta por la transición verde sea contundente y accesible.

- Los esfurzos económicos y operacionales que implican, a corto plazo, justifican la existencia de fondos europeos para sufragar los posibles gastos que se generen. Impulsar proyectos de este tipo va a permitir que exista una mayor claridad para su puesta en marcha y facilitará su implementación.

\subsubsection{Proyectos ligados a la recuperación del medio rural}

Los planes destinados a la reactivación de la actividad económica de las zonas rurales que componen la que se conoce como España vaciada, cuentan con el apoyo de los fondos europeos y de las previsiones señaladas en la Agenda España Digital 2025. Es por ello que, resulta de suma relevancia abordar la modernización de las infraestructuras y contrucciones en estas zonas con el fin de desconcentrar las ciudades permitiendo reactivar la vida rural $e$ incluso creando modelos de producción que apuesten por la descentralización y sean más flexibles. El objetivo de que los alumnos consideren este hecho, pasa por lograr crear una igualdad de oportunidades en el mercado laboral y lograr contribuir del mismo modo a una correcta distribución de la riqueza con nuevas oportunidades, que se generen en zonas que antes eran impensables, dada la escasa inversión de las Administraciones en su impulso.

\subsubsection{La brecha de género y el rol de la Educación Superior}

La búsqueda por construir estrategias que pretendan la búsqueda de nuevas técnicas de implementación en el aula no deben descuidar la importancia de aquellos que dedican parte de su labor al sector de la industria y la construcción. De forma autónoma o complementaria con los alumnos/as del Grado en Derecho, los aprendices del Grado en Eficación deberán proponer estrategias tecnológicas que permitan convertirse en la oferta más viable desde un punto de vista sostenible y económico, sin olvidar las virtudes que ofrecen las nuevas herramientas tecnológicas. Con todo, no son pocos los estudios que demuestran la brecha de género en las carreras ténicas respecto a las que guardan relación con las ciencias sociales y las humanidades. Entre ellos, el Gobierno de España señala la necesidad de, "abordar la cuestión de la igualdad de género en la creación de start-ups, puesto que tan solo el $15,6 \%$ de las start-ups españolas fueron fundadas por mujeres" [11]. Para lograr reducir las barreras de género se pretede buscar técnicas que inciten a que el sector femenino observe a estas carreras académica el mismo interés que presta el sector másculino. Al fin y al cabo, la tecnología constituye de forma transversal el futuro que no deberá entenderse desde la desigualdad de oportunidades en el ámbito: laboral y educativo. Por ello, la realización de la presente práctica deberá considerar los criterios de igualdad por razón de género. Cualquier propuesta positiva que se realice en este sentido, será considerada de forma positiva y al mismo tiempo estaremos atendiendo a uno de los objetivos señalados en la Agenda 2030. 


\subsection{Técnicas específicas de innovación docente para la exposición de proyectos tractores}

Aunque la intención primigenia trata de aunar los esfuerzos y los conocimientos de dos ciencias del saber heterogéneas (jurídicotécnica), observamos que el trabajo colaborativo entre ellas puede resultar positivo. $\mathrm{Si}$ bien es cierto que, aunque la valoración cuenta con la previsión de que se trata de un trabajo en equipo, dentro de cada grupo observamos dos modalidades claramente diferenciadas por quienes pertenecen a la vertiente jurídica y los que forman parte de la vertiente técnica. Es importante señalar la confianza que deberán mostrar los compañeros hacia los conocimientos que presentan la parte del grupo que no forma parte de su disciplina. Con ello queremos hacerles ver como se desarrolla el trabajo en equipo que trabaja por un interés común frente a los valores que defiende la competitividad, que también serán puestos en práctica respecto a los otros grupos. Señaladas estas pautas, resultaría complejo valorar de forma estricta a un alumno de Derecho: la idoneidad de la calidad de los materiales empleados para la construcción del proyecto, recriminarle el no conocer el uso de plataformas como el AutoCat, que sea desconocedor del denominado efecto flecha, o que conozca que las características de un edificio junto al mar deben diferir de los que se construyan sobre la montaña, por el simple hecho de que el salitre podría ser corrosivo para su estructura. Lo mismo ocurre en sentido inverso.

Los efectos de frustración que generaría sobre el alumno del Grado en Edificación al no saber cuáles son las estrategias de gestión pública, regulación del plan general de ordenación urbana, las políticas públicas establecidas desde Europa para incentivar la transición digital, verde y la resiliencia [12], entre otras materias, provocarían el fracaso de la actividad. Puesto que el objetivo de la tarea es aprender desde el trabajo en equipo las ventajas que presenta la innovación desde el ámbito educativo para conocer y crear estrategias que favorezcan la sostenibilidad en los proyectos que se proponen existirán dos aspectos que deberán ser evaluados con exclusividad en el ámbito que ocupa su respectivo estudio: la calidad del proyecto presentado y la veracidad-viabilidad de los datos propuestos (Grado en Edificación) y la dimensión geopolítica, social y administrativa de las decisiones a adoptar (Grado en Derecho).

\subsection{Técnicas comunes de innovación docente para la exposición de proyectos tractores}

Una actividad que apueste por la innovación deberá atender al momento social y las herramientas que dispone para su implementación en el aula. Se trata de un elemento que se presenta como una de las principales características a tener en cuenta si lo que se pretende es lograr alcanzar el éxito en cualquier ámbito que se plantee. La innovación, bien puede consistir en cambios sencillos que permitan potenciar las capacidades de los alumnos (innovación instrumental), o bien pueden crear cambios fundamentales respecto a modelos precedentes (innovación radical o disruptiva). Es por esta causa por la que los docentes deben promover e impulsar la innovación desde el ámbito educativo a fin de alcanzar dos objetivos: la mejora del servicio y la necesidad para alcanzar la adaptación al cambio social.

La propuesta que deseamos abordar viene inspirada por el discurso que formuló en el año 2017 Mark Scott, en el que asentaba las bases de cómo deberían los docentes preparar a las futuras generaciones de estudiantes. En esta línea, Scott recuerda que las letras y las ciencias, "continuarán siendo los cimientos sobre los que descanse todo el aprendizaje". Hace del mismo modo un especial énfasis en la importancia del aprendizaje de la disciplina, "no para que los estudiantes puedan regurgitar los hechos, sino porque es fundamental para la 
comprensión profunda" y para desarrollar "la capacidad de aprender" [13]. Para dar continuidad al argumento desarrollado en el discurso de Scott, no debemos desatander a los desafíos que ocuparán un lugar cada vez más importante: el uso de internet para crear estrategias docentes e incentivar el debate y el razonamiento crítico de los estudiantes de diferentes disciplinas. En el caso propuesto implicará tanto a los estudiantes de Derecho como los de Edificación. Para lograr estos objetivos, pasaremos a enunciar y desarrollar dos estrategias docentes que pueden ser impulsadas de forma conjunta por los representantes de las dos carreras académicas: la creación de un blog, el desarrollo de una oferta pública ficticia, y la generación de un debate tras la publicación de los proyectos tractores propuestos por los estudiantes.

\subsubsection{Creación de un blog}

Para dar comienzo a esta práctica, deberemos proceder a la creación de un blog que nos permita señalar la ficticia convocatoria pública que deberá guiar los proyectos que más tarde presentaran los alumnos/as. Existen para ello, una multiplicidad de plataformas con las cuales poder materializar esta actividad. Sin embargo, algunas de ellas son onerosas y, en muchas ocasiones, inaccesibles al alumno novel. Por suerte, existen otras que nos permiten contar con diversos recursos online para analizar datos tanto cualitativos como cuantitativos, que permiten llevar a cabo los estudios más importantes sin tener que pagar un software de licencia privada $y$, en muchos casos, sin tener que abonar una cuota por la instalación de su programa. Una de ellas se trata de Wordpress. Por Wordpress debemos entender un software de código libre. Su éxito radica en su flexibilidad. Podemos almacenar desde un simple espacio personal a un comercio electrónico o negocio onq denominado E-commerce. La sencillez de su gestión y su autonomía son destacables puesto que cuenta con un escritorio muy intuitivo para poder acceder a los diferentes contenidos. Su característica de código libre ha permitido que diferentes desarrolladores puedan realizar diferentes extensiones de su software original. A este aspecto lo denominamos plugins ya que permiten extender y ampliar la funcionalidad de WordPress, gracias al empleo de plantillas o también denominadas Themes. De este modo, se ha podido observar como las funcionalidades de esta aplicación se han visto exponencialmente incrementadas en los últimos tiempos. La oportunidad de su manejo se debe a que Wordpress se trata de un sistema de gestión de contenidos que agrupa tres virtudes: i) potente; ii) flexible; y, iii) escalable, que nos permitirá gestionar casi cualquier tipo de sitio web, con una instalación y una configuración muy sencilla. La creación de una página de contenidos Wordpress nos da una doble opción: es.wordpress.com y wordpress.org. Podemos comenzar su exposición por estas vías ya que nos proporciona un hosting gratuito $\mathrm{o}$, dicho en otros términos, de un negocio que consiste en alojar, servir, y mantener archivos para uno o más sitios web. El docente si quiere realizar la actividad con su propio hosting, puede formalizar la migración directamente a wordpress.org.

Con todo, vamos a centrarnos en la explicación del sistema web más sencillo, que es es.wordpress.com. Para su realización, el docente dispondrá del dominio que desee dentro del enlace wordpress.com. Si lo que pretendemos es cambiar este nombre, la táctica para hacerlo es comprar el dominio en Wordpress. Otro de los elementos a tener en cuenta, es la creación de una página web de inicio que se convierte en el cartel de bienvenida a las ofertas públicas que presenten los diferentes grupos en la página web. Como tal, deberá mantenerse con un diseño que sea: i) limpio; ii) ordenado; y iii) atractivo, de modo que cause un atractivo a los estudiantes o potenciales consumidores.

\subsubsection{Ofertas públicas a ciegas}

Cuando los alumnos/as tengan listas las prácticas, previo señalamiento de un plazo 
máximo de entrega, deberán subir la oferta a la plataforma creada por el profesor. La posibilidad de que el resto de compañeros vean los resultados del estudio de los compañeros no se hará visible hasta que no estén depositadas todas las tareas. De no ser así, el resto de grupos podrían observar cuál es la estrategia del compañero e intentar mejorarla, lo cual generaría una desigualdad entre los alumnos. Una vez finalizadas las prácticas, el profesor deberá publicar en la plataforma un resumen de las diferentes propuestas atendiendo a tres factores iniciales: gasto que supone para la empresa, gasto que supone para la adminstración y resultados.

En las tablas, el docente no indicará el nombre del grupo. De este modo, se pretende dar conocer la importancia de la protección de datos de las empresas contratistas sin descuidar la necesidad de hacer pública las diferentes opciones tal y como invita el principio de transparencia. Una vez publicadas las diferentes ofertas, los alumnos deberán plantearse cuáles son las diferentes opciones que dispone el docente, en su papel de Administración, para poder llevar a cabo el proceso de selección. Ellos mismos también podrán observar cuál ha sido la estrategia llevada a cabo por el resto de grupos y la apuesta que han hecho por el mantenimiento de la sostenibilidad en su proyecto.

\section{Ejemplos para la publicación de las tablas por parte del docente}

\section{Elija la mejor opción para su ente público entre las opciones disponibles:}

\begin{tabular}{lccc} 
& \multicolumn{1}{c}{} & \multicolumn{1}{c}{ Ente público } & \\
& Ofertas & Gastos & Resultado \\
\hline Opción 1 & $90.000 €$ & $80.000 €$ & $10.000 €$ \\
Opción 2 & $140.000 €$ & $100.000 €$ & $40.000 €$ \\
Opción 3 & $90.000 €$ & $100.000 €$ & $10.000 €$ \\
Opción 4 & $70.000 €$ & $70.000 €$ & $0 €$
\end{tabular}

Tabla 4. Tabla de ingresos y gastos de un ente público 
Innovación docente y sostenibilidad en el aula Belén Andrés Segovia

Vuelva a plantearse la cuestión respecto a los datos agregados a la siguiente tabla:

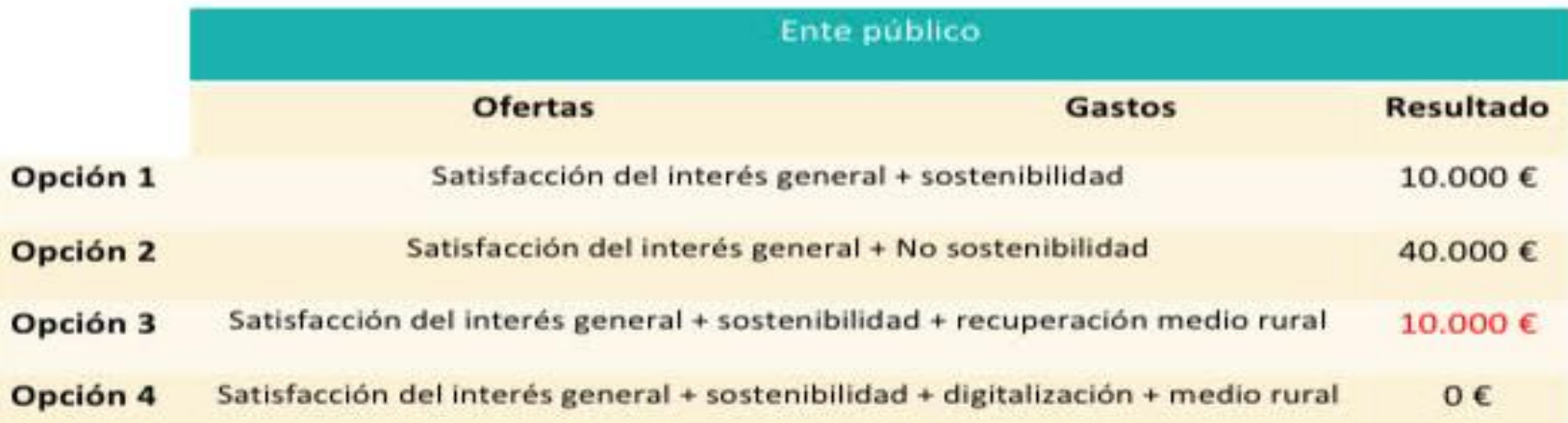

Tabla 5. Tabla de ingresos y gastos con datos agregados que mejoran el servicio

Añadimos un presupuesto financiero, escoja la mejor opción para el ente público:

\begin{tabular}{lrrrr} 
& & \multicolumn{2}{c}{ Ente público } & \\
& Recaudado & Gastado & Resultado & Presupuesto \\
Opción 1 & $90.000 €$ & $80.000 €$ & $1.000 .000 €$ & $70.000 €$ \\
Opción 2 & $140.000 €$ & $100.000 €$ & $4.000 .000 €$ & $170.000 €$ \\
Opción 3 & $90.000 €$ & $100.000 €$ & $1.000 .000 €$ & $100.000 €$ \\
Opción 4 & $70.000 €$ & $70.000 €$ & $0 €$ & $70.000 €$
\end{tabular}

Tabla 6. Tabla de ingresos y gastos con presupuesto financiero

Incorporamos los datos que implica la reforma realizada acorde con las tres E (Eficacia, Economía y Eficiencia):

\begin{tabular}{|c|c|c|c|c|}
\hline & \multicolumn{4}{|c|}{ Ente publico } \\
\hline & Recaudado & Gastado & Resultado & Presupuesto \\
\hline Opción 1 & $90.000 €$ & $\begin{array}{c}80.000 € 80 \mathrm{~m} \text {. de } \\
\text { pavimento a } 1.000 €\end{array}$ & $10.000 €$ & $\begin{array}{l}70.000 € 70 \text { metros de } \\
\text { pavimento a } 1.000 €\end{array}$ \\
\hline Opción 2 & $140.000 €$ & $\begin{array}{l}100.000 € 100 \mathrm{~m} \text {. de } \\
\text { pavimento a } 1.000 €\end{array}$ & $40.000 €$ & $\begin{array}{c}170.000 € 170 \text { metros de } \\
\text { pavimento a } 1.000 €\end{array}$ \\
\hline Opción 3 & $100.000 €$ & $\begin{array}{l}110.000 € 122 \mathrm{~m} \text {. de } \\
\text { pavimento a } 900 €\end{array}$ & $10.000 €$ & $\begin{array}{c}110.000 € 110 \text { metros de } \\
\text { pavimento a } 1.000 €\end{array}$ \\
\hline Opción 4 & $70.000 €$ & $\begin{array}{l}700 € 0,7 \text { metros de } \\
\text { pavimento a } 1.000 €\end{array}$ & $o \in$ & $\begin{array}{l}700 € 0,7 \text { metros de } \\
\text { pavimento a } 1.000 €\end{array}$ \\
\hline
\end{tabular}

Advances in Building Education / Innovación Educativa en Edificación | ISSN: 2530-7940 | http://polired.upm.es/index.php/abe

| Cod. 2103 | Septiembre - Diciembre 2021 | Vol. 5 № 3 | pp. 41/61 | 


\subsubsection{Foro de debate y publicación de la tarea}

Uno de los aspectos que pretendemos fomentar con el presente proyecto es el debate entre los alumnos/as de sendas disciplinas del saber. La ampliación del conocimiento hacia otros campos permitirá conocer nuevas estrategias que les hará más competitivos en el mercado. Aprovechando la creación del blog, los docentes podrán habilitar un apartado de preguntas y respuestas donde los estudiantes plantearan las dudas que les pueda surgir en su desarrollo. Asimismo, se puede disponer de varios botones $u$ opciones para facilitar la entrega de la tarea. A modo de ejemplo, añadir una ubicación, un formulario, una imagen o incluso una encuesta, que permita observar si los alumnos están comprendiendo los objetivos que se marcan.

En la parte derecha del blog podremos crear una serie de aplicaciones que son recomendables señalarlas. La primera pasa porque antes de su publicación es interesante señalar la opción de vista previa con la que podremos visualizar como está quedando nuestro proyecto. También es importante guardar el texto a medida que se va redactando y no publicar hasta que el alumno esté $100 \%$ seguro de que está bien formulado. En esencia, que no falta ninguna documentación, que el texto está redactado sin ninguna falta de ortografía, bien alineado y perfecto para ser publicado. Al mismo tiempo, disponemos de la ventaja que también supone para el docente publicar de forma programada, señalando previamente un horario en función de sus necesidades. Esta es una buena táctica si se encuentra de vacaciones y desea dejar la tarea dispuesta para una fecha concreta. Esta fórmula es una garantía de que se publicará en el horario previsto.

Existen cuatro secciones adicionales dentro de la propia casilla de publicar: 1) formato; 2) categorías; 3) etiquetas; y 4) imagen destacada. En la sección de formato podrán emplear el que más se ajuste al contenido que vayan a defender. Para ello, deberá crear una categoría previa dentro de la sección propia del panel de administrador de blog. En esta localización señalará las categorías temáticas de las que va a constar. De este modo, cuando cree la oferta, éste podrá ir directamente a la categoría que se le haya asignado. También podemos disponer de la sección de etiquetas. Se trata de una herramienta que se asemeja al denominado hashtag de Instagram, donde podrá señalar las palabras que caractericen su propuesta para facilitar la búsqueda de su contenido. Por último, disponemos de la opción de imagen destacada, donde señalaremos el retrato más representativo de nuestro proyecto. Es importante conocer que dentro del cuerpo de nuestra propuesta podremos insertar diferentes instantáneas, pero la imagen destacada se convertirá en la mayor representación de la tarea.

\section{RESULTADOS}

Ampliar los conocimientos hacia nuevas ciencias del saber nunca constituirá un objeto obsoleto. Antes al contrario, permitirá el enriquecimiento de las áreas que convergen en un proyecto común y se verán reforzadas ante los desafíos que presenten las tecnologías avanzadas en el sector de la educación. Para llevar a cabo esta labor, el modelo que proponemos tiene en cuenta las investigaciones realizadas por Carl Benedikt Frey y Michael Osborne sobre la susceptibilidad de las actividades a la información [14]. Dicho esto, la vertiginosidad con la que avanza el cambio tecnológico da cuenta de las implicaciones que esta estrategia docente supondrá sobre las habilidades requeridas, la adquisición de conocimiento y las nuevas formulas de trabajo en equipo. La búsqueda de resultados positivos en este proceso se basa en tres consecuencias: colaboración, inspiración e innovación. Tras anunciar el interés que motivó la creación de esta tarea y el procedimiento metodológico que guía su implementación en el aula, procedemos a señalar a continuación los resultados obtenidos de esta experiencia. En concreto, 
señalaremos: los desafíos, las oportunidades de su implementación y los diferentes puntos de vista y visiones del personal académico en relación con la sostenibilidad en el aula.

\subsection{Desafíos para la implementación de la sostenibilidad en el aula}

Entre los desafíos con los que se topan los docentes del siglo XXI cabe destacar la defensa por un aprendizaje profundo para todos los estudiantes, no sólo para las máquinas. En este sentido, los maestros, desde una escala mundial, deberán reconocer la especial relevancia que adquiere en el proceso educativo la observancia de los retos sociales a los que desde la escuela se puede comenzar a dar solución. La estrategia se basa así en tres fundamentos: lectura, escritura y aritmética. Sin estos elementos, el aprendizaje quedará obsoleto, puesto que dar la espalda a la realidad impedirá que los alumnos entiendan el escenario laboral, social, cultural y político al que se enfrentan. En esencia, la simple adquisición de conceptos teóricos en un aula no son suficientes para los desafíos que plantea el complejo mundo de hoy.

La opción de converger los esfuerzos entre dos disciplinas tan heterogéneas, como son el Grado en Edificación y el Grado en Derecho, no hace más que aceptar la necesidad de establecer un sistema de alfabetización básica suficiente que permita: por un lado, adquirir los conocimientos que sean suficientes para entender como las máquinas van a revolucionar el mercado, haciéndolo con: rapidez, precisión, con el tiempo con un bajo coste y, por otro lado, examinar cómo la adquisición de conocimientos de índole jurídico puede ayudar a que la gestión de esa implementación sea adecuada a los fines perseguidos desde una escala política y social, sin incurrir en ninguna manifestación contraria a derecho.

Los profundos cambios que se avecinan en este campo exigen que exista un enfoque educativo que permita mejorar las competencias de todos los estudiantes. Como en su tiempo expresó Dylan William, "nuestro mundo se está volviendo cada vez más complejo, por lo que se necesitarán niveles cada vez más altos de logros educativos para tener el control de la propia vida, comprender la propia cultura, participar de manera significativa en la democracia $y$ encontrar un trabajo satisfactorio" [15]. Esta afirmación se traduce en las siguientes consecuencias. La existencia de diversos factores en juego, entre los que cabe destacar el papel de los docentes al proporcionar no solo los conceptos teóricos que se requiere por la guía docente de la materia a cursar sino complementar estos términos con la construcción de un marco de comprensión profunda sobre la realidad práctica en la que serán de aplicación. De este modo, los estudiantes podrán aprender a: aplicar los conocimientos adquiridos en el aula de forma creativa y efectiva, reforzar sus estrategias competitivas y colaborativas a través del trabajo en equipo y convertirse en verdaderos pensadores críticos de la realidad que les rodea y que afecta a la sociedad en la que viven. Tal como señaló Richard Watson, "deberíamos darles la confianza y las habilidades para cuestionar la sabiduría convencional y resolver problemas fluidos $y$ conectados, todo lo cual se traduce en enseñar a las personas a pensar por sí mismas" [16]. Por todo ello, la labor del docente, no solo se basará en transmitir su conocimiento al potencial estudiante sino que además le debe acompañar en su proceso de aprendizaje, mostrándole el modo de encontrar y darle sentido a los conceptos teóricos adquiridos. En suma, no deberá circunscribir sus clases al simple dominio de una lista de habilidades.

\subsection{Oportunidades para la implementación de la sostenibilidad en el aula}

La creación de prácticas que permitan concienciar sobre el impacto ambiental que cualquier decisión técnica o jurídica adopte en la construcción de proyectos tractores será determinante para lograr construir un entorno que sea favorable al medio ambiente. Por todo ello, existen cinco razones fundamentales que justifican la propuesta de esta tarea: liderazgo ilustrado; conciencia del riesgo; cambio de 
comportamiento; activismo; y la erradicación de la brecha de género.

A. Liderazgo ilustrado. Tanto docentes como alumnos se encuentran a la vanguardia de la oportunidad que suponen los nuevos medios digitales para lograr incentivar la lucha contra el cambio climático. En estos aspectos, resulta muy importante que los estudiantes sean conscientes que sus decisiones no deben desaprovechar la oportunidad que nos brinda este periodo de crisis sanitaria y los fondos europeos para la recuperación económica. El convencimiento en el que se basa la redacción de estos planes es lograr impulsar a corto plazo, la creación de infraestructuras que serán de aplicación por las generaciones futuras en las próximas décadas. El razonamiento por el que se crea esta práctica pasa por el convencimiento de que la integración de los conocimientos jurídicos con los técnicos supondrán un avance positivo. Por lo que respecta a los alumnos del Grado de la Edificación, deberán velar y apostar por la creación de energías limpias que a su vez, supondrán: un gran atractivo para la generación de empleo, el crecimiento económico y lograrán que los sistemas energéticos se modernicen. Todo ello, sin descuidar su mayor grado de resiliencia y su implicación por alcanzar una menor contaminación [17]. Por otro lado, los alumnos del Grado en Derecho deberán señalar aquellos aspectos que las Administraciones públicas deberán incentivar a fin de estimular los compromisos ecológicos. A modo de ejemplo, señalar ventajas fiscales para aquellos proyectos que apuesten por la descarbonización del sector del transporte, otorgar subvenciones a aquellos proyectos tractores que ofrezcan construcciones que favorezcan la generación de energías limpias, etc.

B. Conciencia del Riesgo. El advenimiento de la pandemia nos hizo más conscientes del papel que ocupaba el sector de la educación para poder buscar sinergias entre los diferentes sectores productivos con el fin, no solo de seguir garantizando el servicio sino de mejorar algunos de los elementos que proponía el modelo anterior. Sin embargo, en este camino hacia la sostenibilidad, la innovación nos hizo ser conocedores de algunos de los riesgos a los que nos enfrentamos colectivamente. Este hecho nos recuerda a que los retos son globales y los ciudadanos se encuentran estrechamente interconectados por nuevas tecnologías que favorecen la interconectividad de todos los agentes. Es por ello que, los alumnos/as deben ser conscientes de los riesgos que suponen sus decisiones, no solo a nivel empresarial sino también respecto al entorno que les rodea. Tras unos meses que han ocupado nuestra preocupación por la pandemia, comienzan a surgir otras cuestiones como son la calidad del aire que respiramos. Es por esta causa por la que observamos como beneficiosa la creación de esta práctica que induce a la comprensión colectiva sobre cómo el tiempo juega en nuestra contra en la lucha por detener las peores consecuencias del calentamiento global y el cambio climático. Este hecho ofrecerá tras sí, la consecución de nuevos retos y la materialización de cambios colectivos e individuales.

C. Cambio de comportamiento. Las actitudes sociales y la demanda han evolucionado de forma favorable hacia la sostenibilidad [18]. El aprendizaje adquirido, tras un periodo de confinamiento causado por la crisis pandémica, nos ha hecho reflexionar sobre la necesidad de llevar una vida más ecológica y saludable. Podríamos advertir que el hecho de vernos compelidos a trasladar las clases magistrales presenciales del aula a formatos virtuales nos ha hecho entender como una mejora del medio ambiente supone a su vez un aumento del bienestar individual. A modo de ejemplo, algunos docentes que habitaban en ciudades diferentes a la sede principal de su centro de trabajo han dejado de consumir medios de transporte que tenían un impacto negativo en el medio ambiente. Quizá sea un simple caso de las enormes consecuencias que este hecho ha podido producir en la sociedad pero supone el inicio de un cambio de mentalidad adquirida que permitirá transformar la forma que tanto docentes como alumnos habían adquirido 
respecto a su forma de trabajar, consumir e invertir parte de su tiempo y esfuerzos en el proceso de aprendizaje.

D. Activismo. Como hemos podido avanzar, la tendencia social hacia la sostenibilidad es en la actualidad un hecho que podemos ver no solo en las aulas, sino en la esfera empresarial y política. Por un lado, pongamos por caso, a nivel empresarial, el caso de BP que durante 2020 hizo una clara apuesta por invertir parte de sus recursos en energías más limpias [19]. También ha adoptado esta senda el gigante de internet Microsoft, apostando y comprometiéndose a una bajada de los índices de carbono. Por otro lado, desde la perspectiva política, encontramos la iniciativa impulsada por la Unión Europea depositada en el Pacto Verde Europeo, que se traduce en una oportunidad para que las autoridades públicas hagan de la Unión Europea un continente líder en alcanzar la neutralidad en las emisiones de carbono. En consecuencia, la dirección hacia esta tendencia esta clara pero, en última instancia el cambio sistémico no solo descansa sobre empresas privadas, públicas o sobre los diferentes fuerzas políticas sino que pasa por nosotros mismos.

E. La brecha de género. Uno de los mayores retos a los que se enfrenta un mundo cada más digitalizado pasa por romper las barreras de género existentes en sectores 0 ámbitos educativos que guarden relación con estrategias técnicas. Es un hecho constatado por diversos estudios el que demuestra que la participación del sector femenino en carreras académicas, que guardan relación con aspectos tecnológicos, es muy inferior al grupo que ocupan las mujeres respecto a los hombres [20]. En un escenario donde la tendencia está marcada hacia la transición digital y la sostenibilidad, esta realidad constituye un factor a tener en cuenta. Esto se debe a que la elección académica determinará el futuro laboral de quienes participen en el mercado. Es por ello, que el estudio de estrategias básicas en este sector, así como la apuesta de las mujeres por este ámbito que se encuentra en ebullición, supondrá no solo una mejora individual sino también social.

\subsection{Puntos de vista y visiones del docente en relación con la sostenibilidad en el aula}

La posición que adoptará el docente en el desarrollo de esta tarea es fundamental al mismo tiempo que se torna algo compleja. En concreto, se le otorga la labor de mediar y guiar las necesidades que plantean los estudiantes en la elaboración del caso práctico. Para ello, deberá tener unos conocimientos previos, no solo sobre la disciplina que le ocupa sino también sobre la asignatura de la carrera académica con la que está colaborando. Este pequeño obstáculo no deja de ser un sucinto reto académico para el mismo pero a su vez constituye una oportunidad en su formación. Los estudiantes, no son el único colectivo que aprende en el transcurso del curso académico sino que el propio maestro se convierte en receptor activo de experiencias y nuevos saberes que compartirá con sus alumnos. Por ello, considero que la realización de esta práctica tiene un beneficio bidireccional. Por un lado, el alumno aprende sobre la importancia de: la sostenibilidad, la regulación y el empleo de las nuevas tecnologías para su futuro laboral y, por otro lado, el docente se nutre de los conocimientos de otras áreas que permitirán consolidar sus investigaciones, apoyándolas en argumentos más fieles al área del conocimiento a la que afecta.

\section{CONCLUSIONES}

Empecemos por el presente y miremos al futuro. Los menores que hoy tiene la temprana edad de cinco años, que comenzaron el parvulario este año, estarán en la universidad en 2030, año previsto por las Naciones Unidas para el cumplimiento de los Objetivos de Desarrollo Sostenible, y pasarán la mayor parte de su vida laboral en la segunda mitad del siglo XXI. Si bien siempre ha sido un hecho constatable que nuestras escuelas sostienen el 
futuro dentro de sus aulas, el sistema educativo actual debe sentar las bases para que estos niños pequeños prosperen en la vida y el trabajo en 2050 y quizás hasta 2090. Tal es el ritmo de cambio producido por el avance de las tecnologías, que es muy concebible que estos menores vivan en un mundo que difiere radicalmente del actual. Eso significa que pensar y planificar el futuro es más importante ahora que nunca.

En estos aspectos, el progreso tecnológico se observa como una fuerza extremadamente poderosa que ha venido para quedarse. No obstante, el impacto que adquirirá sobre el conjunto de la ciudadanía no es el único destino que persiguen. Su implementación no es consecuencia de una utopía ni nos lleva a un futuro no deseado. El poder de cambiar las estrategias educativas se encuentra más bien, en el rol que adquirirán los docentes y los estudiantes en el proceso educativo. En resumidas cuentas el poder para la innovación reside en el ser humano. Por lo tanto, debemos partir de la base de que la innovación docente se observa como una estrategia que podrán emplear los docentes en sus clases con el objeto de atraer la atención de un alumno que vive inmerso en las capacidades que ofrece internet en sus diferentes facetas de desarrollo.

La Covid-19 ha potenciado esta labor, provocando la necesidad de adaptar las clases magistrales a nuevos formatos más digitales. Es probable que la amenaza que propone esta crisis sanitaria no dure para siempre. Sin embargo, la problemática que plantea el cambio climático y los episodios meteorológicos que se han sucedido en los últimos tiempos, dan a conocer la importancia de atender a los factores que guarden relación con la sostenibilidad desde el presente y con vistas a una necesidad que nos acompañará en un futuro. Aunque el riesgo climático se está desarrollando de forma sigilosa, respecto a la pandemia, sus consecuencias pudieran ser más graves. Su persistencia dependerá de la respuesta e implicación que adoptemos, desde los diversos sectores económicos, de cara a detener su expansión.
Por ello, cada medida destinada a reavivar la actividad económica, como en el caso que proponemos de construir una oficina de turismo en una zona marcada por el despoblamiento rural, tendrá un efecto inmediato en nuestra forma de afrontar el bienestar de las futuras generaciones. A su vez, supondrá un impacto ambiental positivo en todo el mundo, que será valorado favorablemente por las instituciones internacionales, europeas y nacionales. En definitiva, es importante que seamos conscientes de las ventajas y los riesgos que supone la sostenibilidad en nuestra sociedad. Por ello, el objetivo de estas lecciones académicas es plantear, desde el empleo de herramientas digitales, cómo estamos y cómo debemos actuar, con el fin de lograr fomentar: el pensamiento crítico y el trabajo en equipo.

\section{AGRADECIMIENTOS}

Sirvan estas últimas líneas para extender mi agradecimiento a quienes desde mis inicios me inculcaron la pasión por la enseñanza y la innovación docente. Entre ellos, a los que confiaron en mí para seguir desarrollando mis inquietudes académicas a través de proyectos y grupos de investigación que siguen vigentes en el momento de presentación del estudio. En concreto, al grupo de investigación INNOVAP (Dret Públic i Innovació) coordinado por el Dr. D. José Luis Blasco Díaz, de la Universitat Jaume I de Castellón (Código de grupo 208). A la Dra. Dña. María Luisa Gómez Jiménez, a través del proyecto, "Sensibilización del alumnado y fomento de la proyección social de la sostenibilidad desde el empleo de herramienta innovadoras de internacionalización curricular: Consolidando el Grupos RISPINES de Innovación Docente", (ref. RISPINES-LAB PIE 19-153). Al Dr. D. José Carlos Pedrosa López, en su proyecto de innovación docente RENOVAPID para el curso 2021/2022 de la Universitat de València: "Consolidación académica del blog jurídico online en la década digital" (ref. UV-SFPIE_PID1639324). Y, por último, al Dr. D. Pedro Chaparro Matamoros, por su proyecto de 
innovación docente RENOVAPID para el curso 2021/2022 de la Universitat de València: "Juega y aprende: La ludoteca jurídica 2.0" (ref. UVSFPIE_PID-1641711). Estoy convencida de que el éxito de la innovación docente, en los centros de Enseñanza Superior, con personas tan válidas como las citadas está garantizado.

\section{REFERENCIAS}

[1] Este estudio se ha realizado en el marco de los siguientes grupos y proyectos de investigación: "INNOVAP (Dret Públic i Innovació)" grupo de investigación coordinado por el Dr. D. José Luis Blasco Díaz, de la Universitat Jaume I de Castellón (Código de referencia del grupo 208); "Sensibilización del alumnado y fomento de la proyección social de la sostenibilidad desde el empleo de herramienta innovadoras de internacionalización curricular: Consolidando el Grupo RISPINES de Innovación Docente", (ref. RISPINES-LAB PIE 19-153). IP: M.L. Gómez Jiménez. Universidad de Málaga; el proyecto de innovación docente RENOVAPID para el curso 2021/2022 de la Universitat de València: "Juega y aprende: La ludoteca juridical 2.0" (ref. UV-SFPIE_PID-1641711). IP: P. Chaparro Matamoros; y el proyecto de innovación docente RENOVAPID para el curso 2021/2022 de la Universitat de València: "Consolidación académica del blog jurídico on-line en la década digital" (ref. UV-SFPIE_PID-1639324). IP: J.C. Pedrosa López.

[2] Naciones Unidas, Memoria del Secretario General sobre la labor de la Organización, 2015: https://undocs.org/es/A/70/1.

[3] Naciones Unidas, Informe de los Objetivos de Desarrollo Sostenible 2020: https://unstats.un.org/sdgs/report/2020/TheSustainable-Development-Goals-Report2020_Spanish.pdf.
[4] M. Tucker, "Educating for a Digital Future: Notes on Curriculum" Education: Future Frontiers Occasional Paper Series, NSW Department of Education, June.

[5] World Economic Fórum, Global Risks, 2021: http://reports.weforum.org/global-risks-report2021/methodology/

[6] Comisión Europea, Comunicación de la Comisión al Parlamento Europeo, al Consejo, al Comité Económico y Social Europeo y al Comité de las Regiones relativa a la consecución del Espacio Europeo de Educación de aquí a 2025 \{SWD(2020) 212 final\}, Bruselas, 30.9.2020, $\operatorname{COM}(2020) 625$ final.

[7] Comisión Europea, Comunicación de la Comisión al Parlamento Europeo, al Consejo Europeo, al Consejo, al Comité Económico y Social Europeo y al Comité de las Regiones, El Pacto Verde Europeo, Bruselas, 11.12.2019, COM(2019) 640 final.

[8] Comisión Europea, Plan de recuperación para Europa, 2020: https://ec.europa.eu/info/strategy/recovery-planeurope_es.

[9] Gobierno de España, Agenda España Digital 2025, Ministerio de Asuntos Económicos y transformación 2020: https://portal.mineco.gob.es/caes/ministerio/estrategias/Pagines/00_Espana_Di gital_2025.aspx.

[10] K. Schwab, La cuarta revolución industrial, Penguin Random House Grupo Editorial, Barcelona, 2016.

[11] Gobierno de España, Agenda España..., op.cit. 2020.

[12] Comisión Europea, EU budget: European Commission welcomes agreement on $€ 1.8$ trillion package to help build greener, more digital and more resilient Europe, Bruselas 10 de noviembre de 2020: https://ec.europa.eu/commission/presscorner/d etail/en/IP_20_2073. 
[13] M. Scott, Preparating today's students for tomorrow's world, NSW Departamento de Educación, 2017: https://education.nsw.gov.au/teaching-andlearning/education-for-a-changingworld/resource-library/preparing-today-sstudents-for-tomorrow-s-world.

[14] C. Benedikt Frey and Michael A. Osborne, The Future of Employment: How susceptible are jobs to computerisation?, September, 2013:

https://www.oxfordmartin.ox.ac.uk/publications/t he-future-of-employment/.

[15] D. Wiliam, "Wale Pisa Results: 'Little will be learned'", BBC News, 4 December 2016.

[16] R. Watson, "On education in the $21^{\text {st }}$ Century", Education Future Frontiers Occasional Paper Series, NSW, Department of Education, June 2017.

[17] D. Jørgensen y F. Birol, "How clean energy transitions can help kick-start economies", Agencia Internacional de la
Energía, 23 de abril de 2020: https://www.iea.org/commentaries/how-cleanenergy-transitions-can-help-kick-starteconomies.

[18] K. Schwab y T. Malleret, Covid-19: El gran reinicio, Forum Publishing, Ginebra, 2020. [19] L. Hurst, "Supermajors find obstacles, and opportunities, as pandemic drags on", World Oil, 16 de junio de 2020: https://www.worldoil.com/news/2020/6/16/super majors-find-obstacles-and-opportunities-aspandemic-drags-on.

[20] Ministerio de Educación y Formación profesional, Igualdad en cifras MEFP 2020: Aulas por la lgualdad, Madrid, 2020: https://www.educacionyfp.gob.es/dam/jcr:914e 956e-9241-49c5-b9a6-d99d6eade751/igualdaden-cifras-2020-online.pdf. 